Research Article

\title{
Epigenetic Effects of Micronized Matrix Allograft Cartilage on Stem Cell Differentiation
}

\author{
Timothy Reed, $B S^{1,2}$, Taylor Mustapich, $B A^{2,3}$, Brandon Alba, $M D^{1,2,4}$, Haixiang Liang, $M D^{2}$ and Daniel $A$ \\ Grande, $P h D^{1,2,5^{*}}$
}

${ }^{1}$ Donald and Barbara Zucker School of Medicine at Hofstra/Northwell, Hempstead, NY, USA

${ }^{2}$ Orthopaedic Research Laboratory at Feinstein Institute for Medical Research, Northwell Health, Manhasset, NY, USA

${ }^{3}$ Albert Einstein College of Medicine, Bronx, NY, USA

${ }^{4}$ Rush University Medical College, Chicago, IL, USA

${ }^{5}$ Department of Orthopaedic Surgery, Northwell Health, Great Neck, NY, USA

\begin{abstract}
Objective: Articular cartilage injuries can lead to significant joint morbidity. Although marrow stimulation procedures such as microfracture are a popular treatment option, results are often suboptimal, with resultant neocartilage exhibiting a more fibrous than hyaline phenotype. Micronized matrix allograft cartilage has emerged as a promising adjunct to augment microfracture procedures by promoting a greater degree of hyaline cartilage regeneration and improved longterm outcomes. However, little is known about the effects of micronized matrix allograft cartilage on a cellular level. The purpose of this study was to investigate the epigenetic effects of micronized matrix allograft cartilage on multipotent stem cell differentiation.
\end{abstract}

Design: Human mesenchymal stem cells (MSCs) were treated with BioCartilage (micronized matrix allograft cartilage; Arthrex Inc., Naples, FL) and/or human platelet-rich plasma (PRP). Chondrogenic gene expression was assessed using qRT-PCR to measure expression of type II collagen (COL2), aggrecan (AGG), and SYR-Box 9 (SOX9) marker genes. PCR was performed on day 3, day 7, and day 14 of treatment. Human adipose-derived stem cells (ADSCs) were also treated with BioCartilage; osteocalcin (OCN), alkaline phosphatase (ALP), and RUNX2 marker genes were used to measure osteogenic gene expression, and CD31 and VEGF were used to measure angiogenic gene expression. PCR was performed on day 14 of treatment.

Results: MSCs treated with BioCartilage resulted in a significant increase in SOX9 expression at all three timepoints. COL2 expression was found to be elevated at the day 7 timepoint. BioCartilage combined with PRP demonstrated a synergistic effect on increasing AGG expression. In the ADSCs, treatment with BioCartilage resulted in a decrease in ALP expression, but did not alter OCN or RUNX2 expression. ADSCs treated with BioCartilage also exhibited decreased CD31 expression and variably increased VEGF expression.

Conclusions: This study supports a mechanism of action by which BioCartilage promotes hyaline cartilage gene expression that may ultimately influence the pro-chondrogenic differentiation of stem cells. The data also suggests that BioCartilage inhibits genes associated with bone formation which is important for maintaining cartilage regeneration in microfracture procedures, and may enhance genes associated with blood vessel formation, which has a more ambiguous role in chondrogenic differentiation.

\section{Keywords}

Micronized matrix allograft, Microfracture, Platelet-rich plasma, Articular cartilage, Mesenchymal stem cells

\section{Introduction}

Articular cartilage injuries are highly prevalent and can cause significant morbidities including pain, joint dysfunction, and progression to osteoarthritis [1-3]. Due to the low regenerative capacity and avascular structure of cartilage tissue, these lesions have a limited capacity to heal spontaneously [4-7]. Thus, surgical intervention is often required. In older
*Corresponding author: Daniel A. Grande, Feinstein Institute for Medical Research, 350 Community Drive, Manhasset, NY 11030, USA, Tel: (516)-562-1138, Fax: (516)-562-1022

Accepted: February 23, 2021

Published online: February 25, 2021

Citation: Reed T, Mustapich T, Alba B, et al. (2021) Epigenetic Effects of Micronized Matrix Allograft Cartilage on Stem Cell Differentiation. Regen Med Ther 4(1):46-54 
Citation: Reed T, Mustapich T, Alba B, et al. (2021) Epigenetic Effects of Micronized Matrix Allograft Cartilage on Stem Cell Differentiation. Regen Med Ther 4(1):46-54

patients, these lesions can be managed effectively with arthroplasty. However, arthroplasty procedures are not ideal for younger patients due to concerns regarding implant durability, residual functional limitations, and an increased likelihood of multiple revision surgeries [2,8-11].

As an alternative to arthroplasty, many surgical interventions aim to treat lesions by stimulating regeneration using endogenous cells, such as mesenchymal stem cells [12]. The most commonly used of these procedures is microfracture [13], a single-stage, low-cost option for treating articular cartilage defects [5]. Microfracture involves piercing the subchondral bone in order stimulate the migration of endogenous stem cells from the bone marrow, which in turn facilitates cartilage regeneration [14]. However, the outcomes of these procedures can be suboptimal. Surgical outcomes are highly dependent on certain patient factors, including body mass index (BMI) and the size and location of the lesion [15]. The neocartilage formed after microfracture is also more fibrous than hyaline in quality, and lacks the biomechanical properties and durability of native articular cartilage [16-19]. Clinical studies have found that following an initial improvement after microfracture, a subsequent decline in patient-reported activity scores was observed in $47 \%$ of athletes, and only $44 \%$ of athletes were able to return to regular participation in high-impact, pivoting sports by 48 months post-operation [20].

To augment the results of microfracture procedures, a variety of adjuncts have been developed. One promising technique is the use of micronized matrix allograft cartilage. Micronized matrices are composed of a dehydrated, acellular form of cartilage that retains the extracellular matrix (ECM) components, thereby acting as a scaffold to stimulate autologous cell interactions [21]. The micronized matrix can be mixed with platelet-rich plasma (PRP) and implanted into a microfracture lesion, then held in place with fibrin glue. Studies utilizing in vivo microfracture in numerous animal models have shown encouraging results with the use of micronized cartilage matrix with PRP [22-24]. However, there is currently a paucity of in vitro studies that have investigated the effects of micronized matrix allograft cartilage at the genetic and cellular levels.

The purpose of this study was to better understand the complex role that micronized allograft cartilage plays in tissue regeneration. Specifically, this study focused on the use of BioCartilage (Arthrex Inc., Naples, FL), a proprietary micronized allograft cartilage product. In vitro experiments using two different cell types were conducted to evaluate the epigenetic effects of this material with the aim of better elucidating its influence on cellular differentiation as a potential target for use in the clinical setting.

\section{Methods}

\section{Chondrogenic gene expression}

Human mesenchymal stem cells (MSCs) were isolated from the bone marrow of donors. The cells were expanded in culture using DMEM/F-12 growth media (Cellgro, Manassas, VA) with $10 \%$ fetal bovine serum (FBS) and $1 \%$ antibiotic-an- timycotic solution (Cellgro). The cultures were incubated in a $5 \% \mathrm{CO}_{2}, 95 \%$ humidity water-jacketed incubator at $37^{\circ} \mathrm{C}$. The cells were collected at passage 3 and seeded in monolayer onto standard 12-well plates. Upon reaching $90 \%$ confluence, the cells were exposed to one of four treatments: 1) Standard growth media only, which served as a control; 2) Media plus $3.5 \mathrm{mg}$ of BioCartilage; 3) Media plus $5 \%$ inactivated human PRP; 4) Media plus BioCartilage and PRP. Quantitative reverse transcriptase polymerase chain reaction ( $\mathrm{QRT}$-PCR) was performed to measure the relative expression of three genetic markers associated with the hyaline chondrogenic phenotype: Type II collagen (COL2), aggrecan (AGG), and SYR-Box 9 (SOX9) [25]. qRT-PCR was performed on day 3, day 7, and day 14 of treatment exposure. One-way analysis of variance [26] was used to compare gene expression among treatment groups and timepoints. $P$ values less than 0.05 were considered statistically significant.

\section{Osteogenic and angiogenic gene expression}

Adipose-derived stromal cells (ADSCs) were isolated from human donors and cultured using standard growth media and incubation protocols as described above for MSCs. At passage four, the cells were harvested and seeded in monolayer onto 12-well plates and exposed to one of two treatments: 1) Osteogenic culture media, containing DMEM/F-12 + 10\% FBS + $100 \mathrm{nM}$ dexamethasone $+200 \mu \mathrm{M}$ sodium ascorbic acid + beta-glycerophosphate, which served as a control group; or 2) Osteogenic culture media plus BioCartilage.

In addition to being cultured in monolayer, ADSCs were also cultured in pellet structure using chondrogenic media (DMEM/F-12 + 1\% FBS + 1\% insulin-transferrin-selenous acid (ITS) supplement $+10 \mathrm{nM}$ dexamethasone $+200 \mu \mathrm{M}$ sodium ascorbic acid $+10 \mathrm{nM}$ transforming growth factor beta3 [TGF- $\beta 3$ ]). After formation of a pellet, the cells were then treated with either osteogenic media only or osteogenic media plus BioCartilage.

Both the monolayer and the pellet groups were analyzed at day 14 using qRT-PCR to measure relative expression of genes associated with an osteogenic phenotype [27], including runt-related transcription factor 2 (RUNX2), alkaline phosphatase (ALP), and osteocalcin (OCN), as well as genes associated with an angiogenic phenotype [28], including vascular endothelial growth factor A (VEGFa) and CD31. $P$ values less than 0.05 were considered statistically significant.

\section{Results}

\section{Chondrogenic gene expression}

SOX9 expression increased with the addition of BioCartilage compared to the control group at all three timepoints (Figure 1A). The addition of PRP had no influence on SOX9 expression at day 3 and day 7. However, the PRP-treated group saw a decrease in SOX9 expression compared to the control group at day 14. The addition of both PRP and BioCartilage resulted in an increase in SOX9 expression at day 3 and day 7 , but not day 14 . Overall, the expression of SOX9 decreased over the course of two weeks in most treatment groups (Figure 1B). However, the control group demonstrated a slight 
Figure 1A.

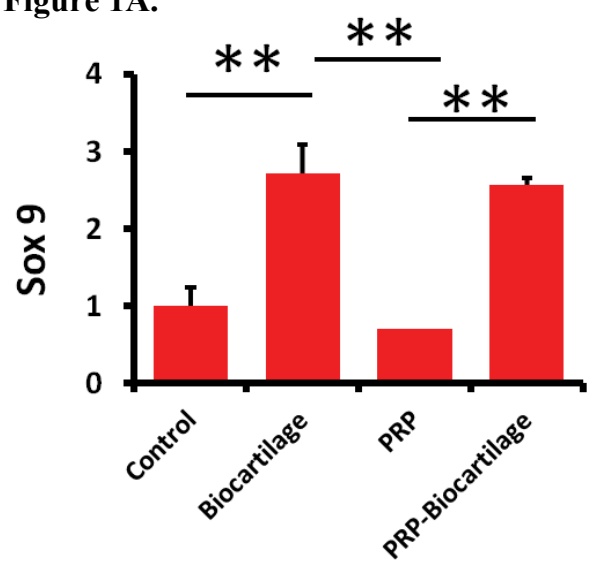

3 days

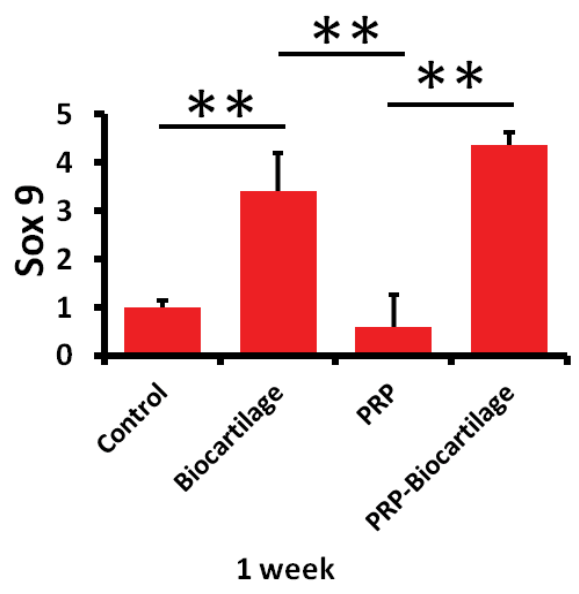

1 week

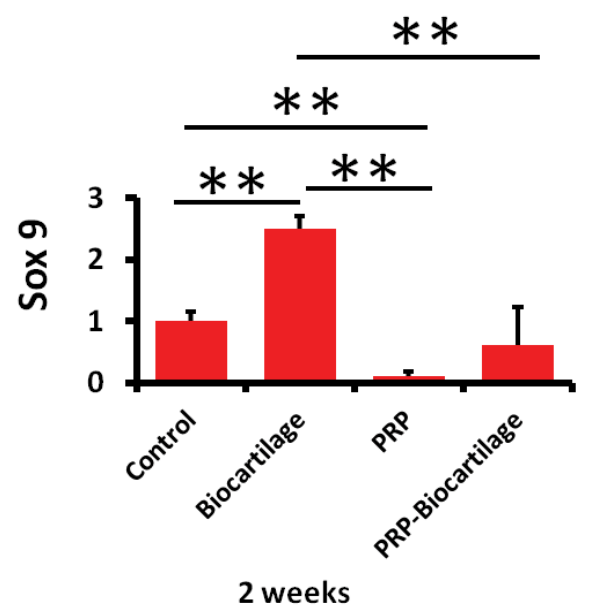

Figure 1B.

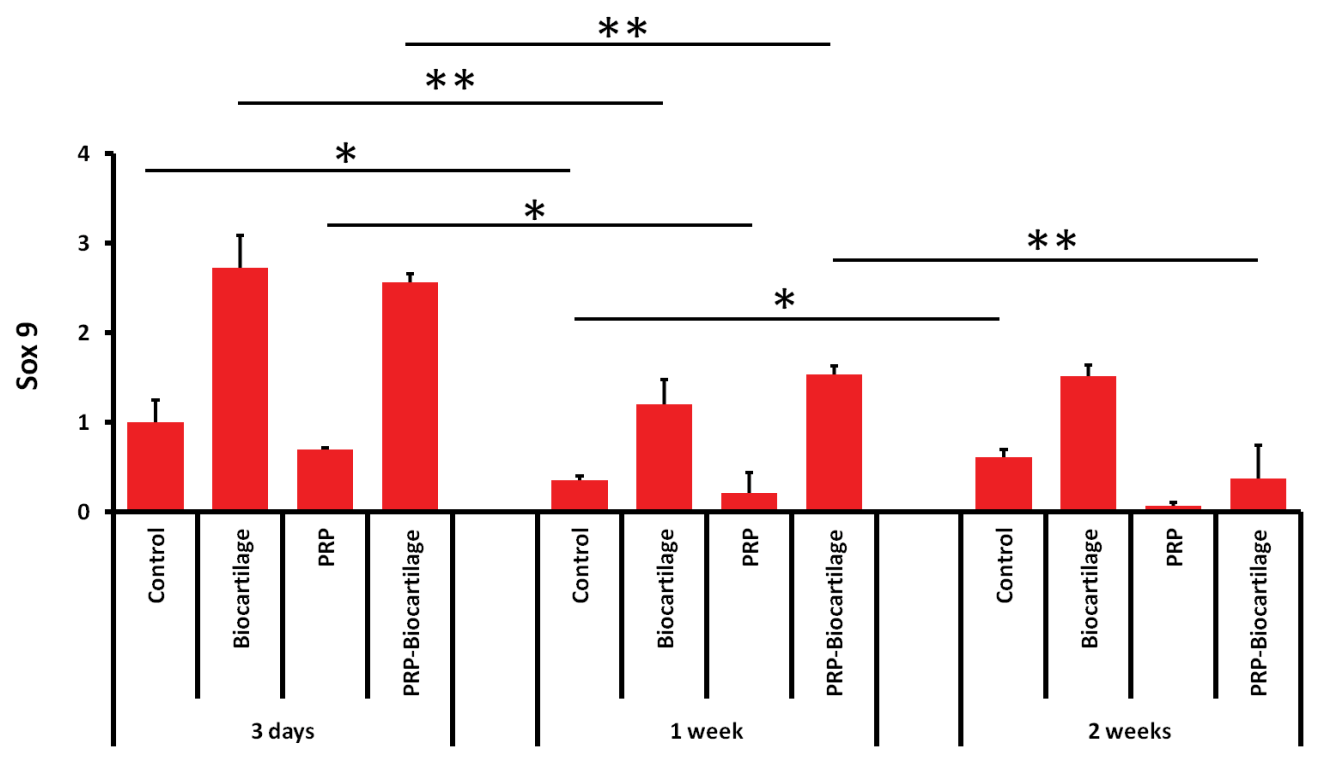

Figure 1: A) SOX9 gene expression compared between treatment groups within each timepoint; B) SOX9 gene expression compared between timepoints.

"denotes $P<0.05$, and ${ }^{* *}$ denotes $P<0.01$. 
Figure 2A.

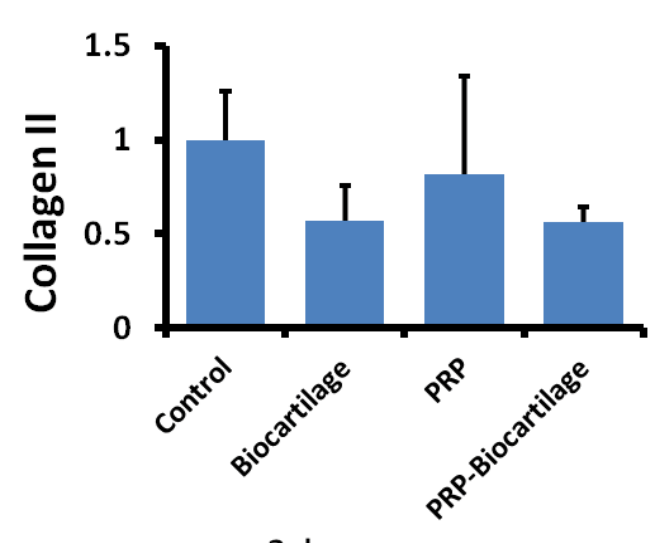

3 days

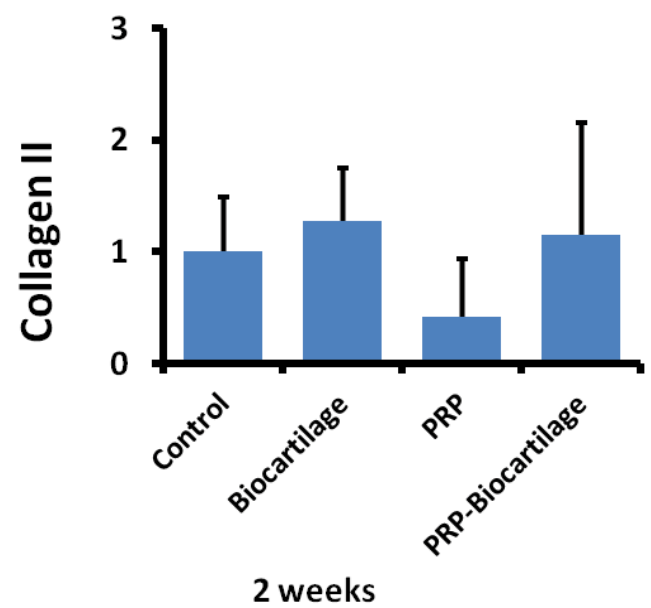

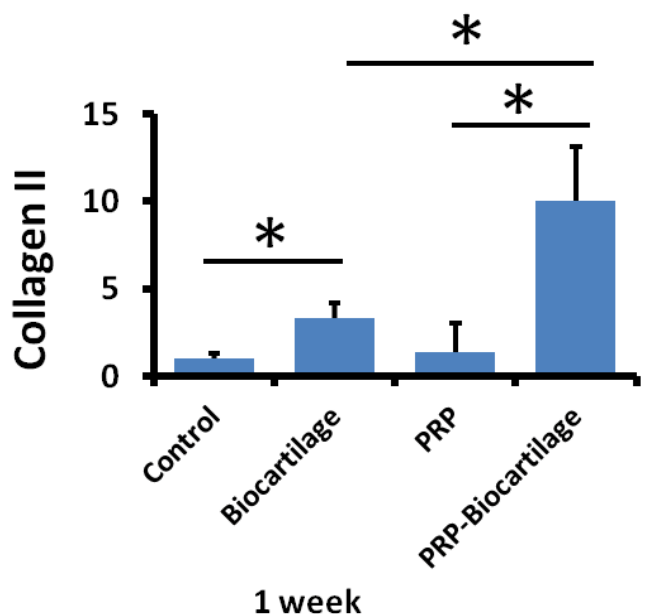

1 week

Figure 2B.

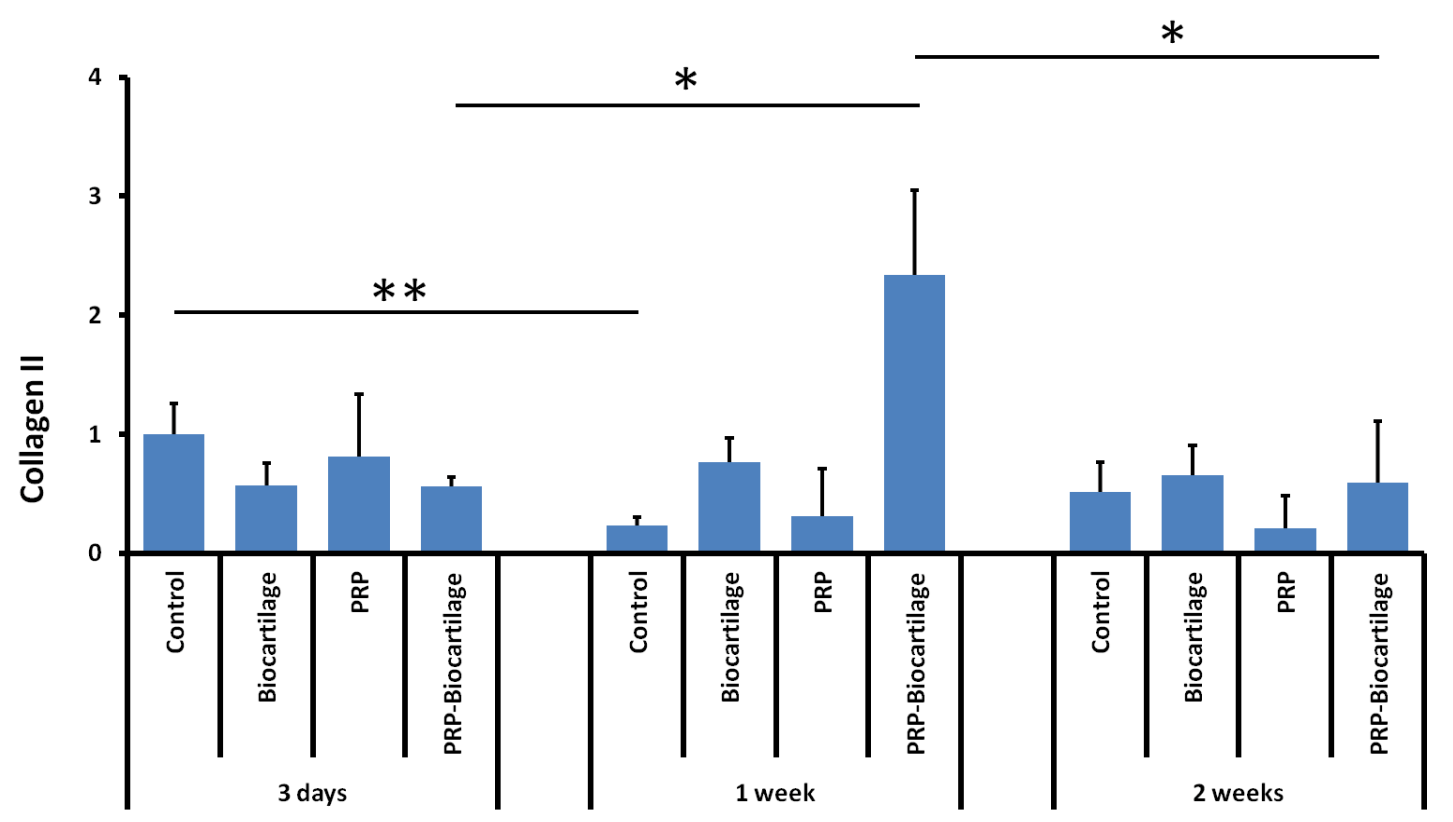

Figure 2: A) COL2 gene expression compared between treatment groups within each timepoint; B) COL2 gene expression compared between timepoints.

"denotes $P<0.05$, and ${ }^{* *}$ denotes $P<0.01$. 
Citation: Reed T, Mustapich T, Alba B, et al. (2021) Epigenetic Effects of Micronized Matrix Allograft Cartilage on Stem Cell Differentiation. Regen Med Ther 4(1):46-54

increase in SOX9 expression between the day 7 and day 14 timepoints.

COL2 expression increased with the addition of BioCartilage at the day 7 timepoint only (Figure 2A). PRP did not significantly affect COL2 expression at any timepoint. At the day 7 timepoint only, the addition of both PRP and BioCartilage significantly increased COL2 expression compared to both the control group and the BioCartilage only treatment group. Overall, most treatment groups maintained relatively similar expression of COL2 over the course of two weeks, except for the control group, which showed a decrease in COL2 expression at the day 7 timepoint (Figure 2B).

The expression of AGG was not significantly affected by the addition of BioCartilage at any timepoint (Figure $3 \mathrm{~A}$ ). The addition of PRP resulted in a decrease in AGG expression compared to both the control group and the BioCartilage group at the day 14 timepoint. The addition of both PRP and BioCartilage resulted in a significant increase in AGG expression compared to all other groups at the day 7 timepoint. However, at the day 14 timepoint, PRP and BioCartilage resulted in a significant decrease in AGG expression compared to both the control group and the BioCartilage group. Overall, AGG expression decreased over time in most groups (Figure $3 \mathrm{~B}$ ).

\section{Osteogenic and angiogenic gene expression}

The addition of BioCartilage did not significantly affect RUNX2 or OCN gene expression in either the monolayer or pellet experimental groups (Figure 4A and Figure 4B). ALP expression decreased with the addition of BioCartilage in both the monolayer and the pellet groups (Figure $4 \mathrm{C}$ ). The addition of BioCartilage resulted in an increase in VEGFa expression in the pellet group, but not in the monolayer group (Figure 4D). BioCartilage also resulted in a decrease in CD31 expression in both the monolayer and pellet groups (Figure 4E).

\section{Discussion}

Articular cartilage injuries are a common cause of significant joint disability. Thus, finding adequate treatment options to improve quality of life for affected patients is imperative. Marrow stimulation procedures are designed to access the mesenchymal cells present in the subchondral bone so that these cells may fill the cartilage defect and differentiate into cartilage tissue. Microfracture is the most commonly employed marrow stimulation procedure, as it is an attractive, low-cost, single stage option for treating articular cartilage defects. However, patients often have residual functional limitations after microfracture, likely related to the fact that the resulting neocartilage is typically more fibrous than hyaline in quality. Researchers are continuously searching for new methods to obtain a more hyaline-like tissue fill of the cartilage defect in order to achieve improved mechanical properties and better long-term outcomes. Micronized matrix allograft cartilage products such as BioCartilage have emerged as a promising new adjunct to microfracture procedures that can augment surgical outcomes. However, little is currently known about the effects of such adjunctive therapies on the cellular level. This study offers important insight into the epigenetic effects of BioCartilage on multipotent stem cells, which in turn may provide a better understanding of how micronized matrix allograft cartilage acts to regenerate cartilage tissue and subsequently improve joint function.

The data from this study demonstrates that MSCs treated with BioCartilage undergo an epigenetic upregulation in SOX9 and $\mathrm{COL} 2$ gene expression at various timepoints. This suggests the MSCs were exhibiting pro-chondrogenic behavior, as SOX9 and COL2 are markers of a hyaline cartilage phenotype. MSCs treated with PRP alone tended to demonstrate a decrease in SOX9 and AGG gene expression, indicating a possible inhibition of cartilaginous differentiation. However, the combination treatment of both BioCartilage and PRP showed a strong synergistic effect on increasing the expression of COL2 and AGG genes at the day 7 timepoint. This implies that the MSCs were undergoing the greatest degree of pro-chondrogenic hyaline gene expression after 7 days of BioCartilage + PRP treatment. It also supports the idea that a combination of BioCartilage and PRP may serve as a powerful means for augmenting hyaline cartilage formation in microfracture procedures.

In the second phase of this study, ADSCs cultured in both monolayer and pellet form were treated with BioCartilage to examine the behavior of both osteogenic and angiogenic genes and to identify possible competition with the chondrogenic pathway. RUNX2, OCN, and ALP were used as marker genes to assess pro-osteogenic behavior. The addition of BioCartilage did not result in any statistically significant difference in RUNX2 or OCN gene expression in the monolayer nor pellet group. However, ALP gene expression was significantly decreased in the BioCartilage-treated groups in both the monolayer and pellet forms. ALP is an important marker of osteogenic differentiation, and these results suggest BioCartilage was downregulating genes associated osteogenic maturation of the ADSCs to some degree.

CD31 and VEGF were used as marker genes to measure the epigenetic influence of the micronized matrix allograft material on angiogenic factors. Treatment with BioCartilage resulted in a decrease in $C D 31$ gene expression in both the monolayer and pellet ADSC cultures. A decrease in CD31 expression, an endothelial cell surface marker used to detect angiogenesis [28], suggests that BioCartilage was acting to suppress angiogenic differentiation and the formation of new blood vessels. Since cartilage tissue is avascular, this is consistent with the idea that BioCartilage promotes chondrogenic differentiation. The data also revealed a significant increase in VEGFa gene expression in the BioCartilage-treated pellet culture compared to the control pellet. VEGFa is a member of the platelet-derived growth factor (PDGF)/vascular endothelial growth factor (VEGF) family and induces angiogenesis and migration of endothelial cells to form new blood vessels. While this initially seems at odds with the suppressed CD31 expression, VEGF has also been found to be a crucial factor in promoting chondrocyte survival and maturation $[29,30]$. Therefore, this suggests that while BioCartilage may inhibit the proliferation and growth of endothelial cells through suppression of CD31, it may also support chondrogenic differentiation of the ADSCs through a promotion of VEGFa. However, this picture is confounded by studies that have shown 
Figure 3A.

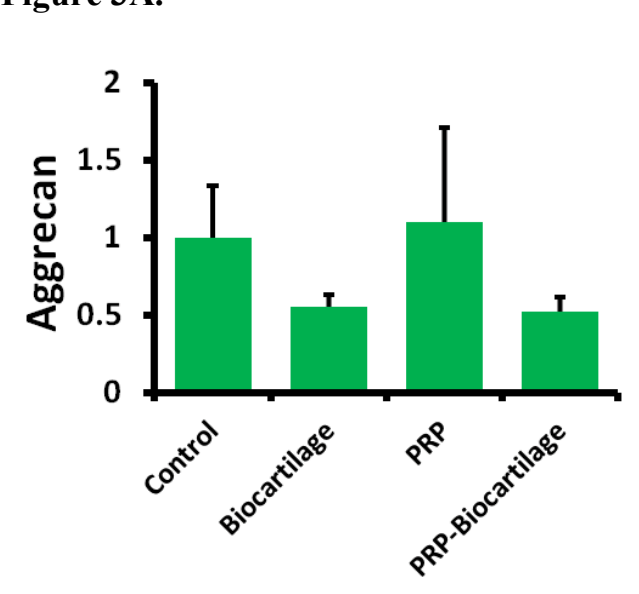

3 days

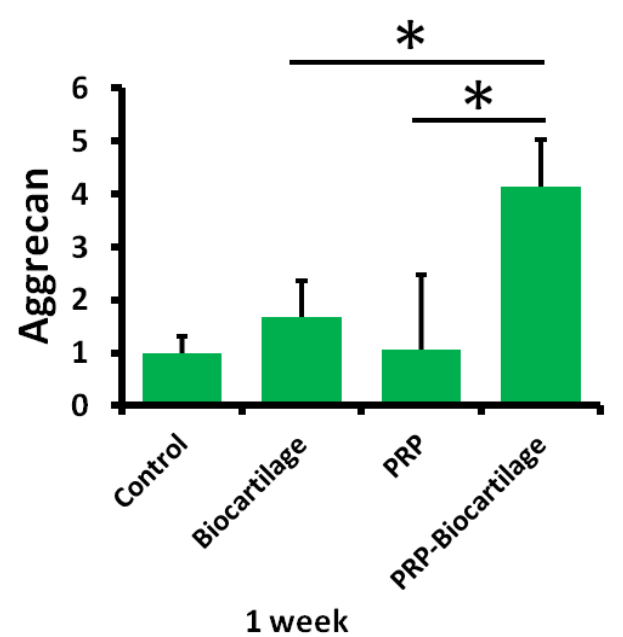

1 week

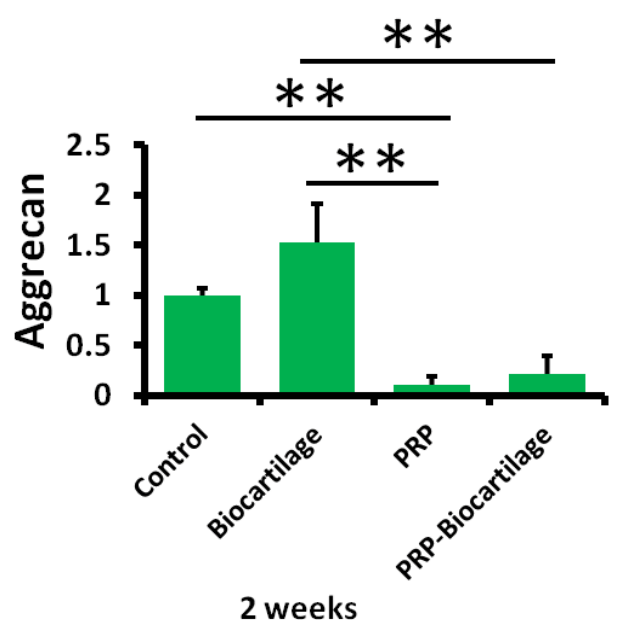

Figure 3B.

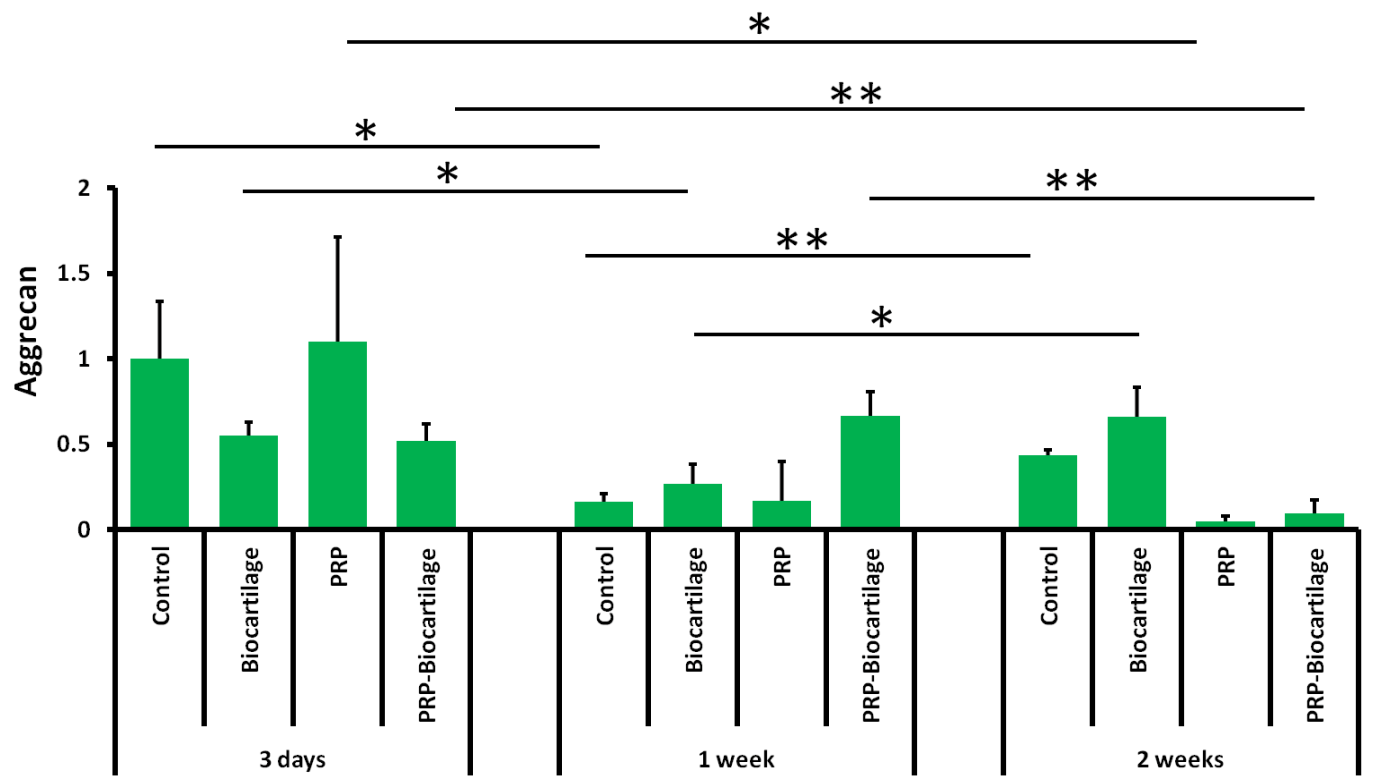

Figure 3: A) AGG gene expression compared between treatment groups within each timepoint; B) AGG gene expression compared between timepoints.

*denotes $P<0.05$, and ${ }^{* *}$ denotes $P<0.01$. 
Figure 4A.
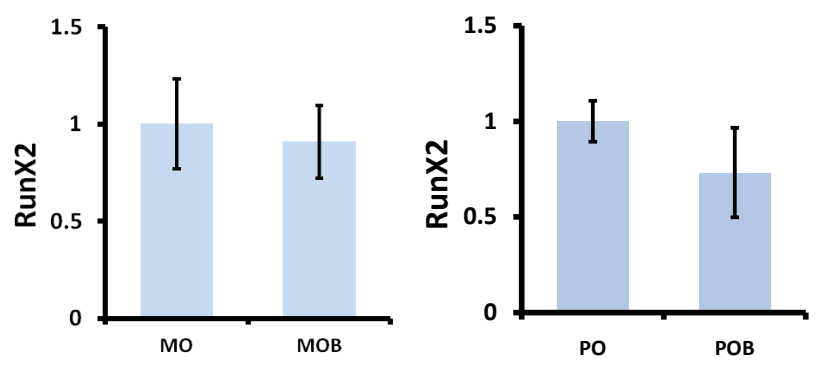

Figure 4B.
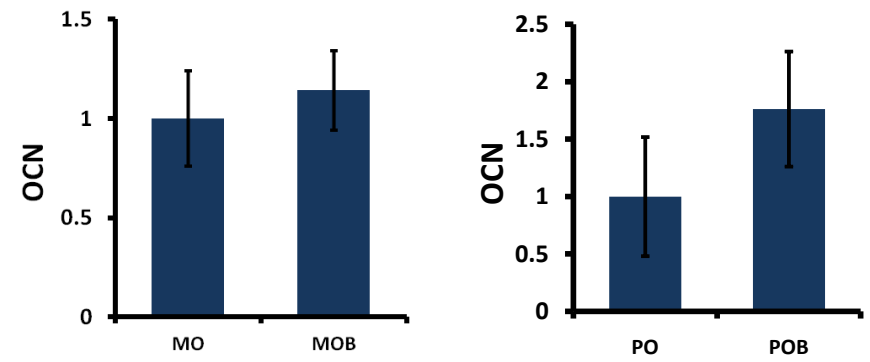

Figure 4C.
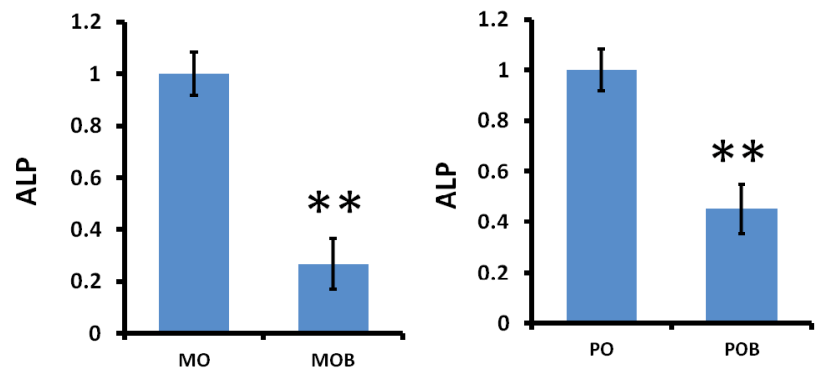

Figure 4D.
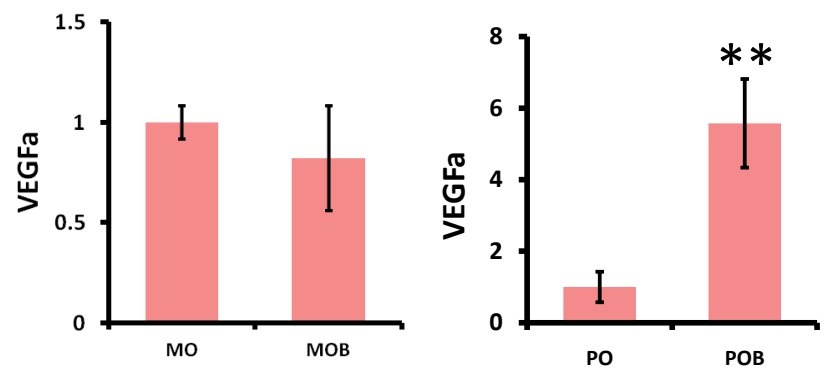

Figure 4E.
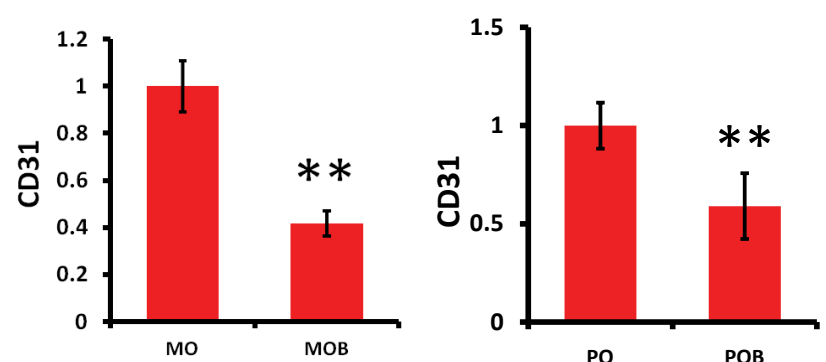

Figure 4: Gene expression at day 14 for monolayer and pellet experimental groups. MO: Monolayer with osteogenic media; MOB: Monolayer with osteogenic media and BioCartilage; PO: Pellet with osteogenic media; POB: Pellet with osteogenic media and BioCartilage. A) RUNX2; B) OCN; C) ALP; D) VEGFa; E) CD31. 
Citation: Reed T, Mustapich T, Alba B, et al. (2021) Epigenetic Effects of Micronized Matrix Allograft Cartilage on Stem Cell Differentiation. Regen Med Ther 4(1):46-54

increased VEGF expression by ADSCS can inhibit chondrocyte phenotype and induce chondrocyte apoptosis [31].

Overall, the results of this study indicate that BioCartilage promotes the epigenetic upregulation of pro-chondrogenic - and more specifically, pro-hyaline - genes by multipotent stem cells. BioCartilage also seems to inhibit pro-osteogenic and pro-angiogenic gene expression and thus presumably differentiation. These conclusions support in vivo animal studies and human clinical trials that report BioCartilage is a powerful adjunct for inducing hyaline cartilage regeneration in microfracture procedures [22-24]. Clinical trials to compare the efficacy of BioCartilage versus standard-of-care treatments of osteochondral defects are currently underway [32,33]. Although relatively few human clinical studies of BioCartilage have been published, the general consensus thus far has been that BioCartilage results in a superior, more hyaline-like cartilage fill that mimics native articular tissue more closely than traditional microfracture procedures alone with virtually negligible risk [34-37].

The authors recognize that this study is not without limitations. Performing qRT-PCR gene analysis at additional timepoints may have provided a higher level of evidence and thus been better able to elucidate the effects of BioCartilage on gene expression over time. Analyzing a greater number of genes may also have allowed this study to better pinpoint the specific effects of BioCartilage and its role in cellular differentiation. Additionally, duplicating the study with ADSCs and MSCs in the chondrogenic and osteo-/angiogenic portions of the protocol, respectively, would allow for a more complete profile of the chondrogenic, osteogenic, and angiogenic gene expression in each of the two stem cell lines and thus a more appropriate correlation of the chondrogenic and osteo-angiogenic behaviors in each individual cell line. Finally, although upregulation of the aforementioned genes is highly suggestive of a pro-chondrogenic influence on stem cells by BioCartilage, future studies will include more definitive quantitative protein studies and histologic evaluation to better validate this hypothesis.

This study provides valuable new information to the existing literature regarding the effects of micronized matrix allograft cartilage on articular cartilage regeneration. Specifically, this study better elucidated how BioCartilage may influence cartilage, bone, and blood vessel differentiation of stem cells on the genetic level. This knowledge affords orthopedic researchers and clinicians a better understanding of how materials like BioCartilage affect the stem cell-rich milieu of microfracture procedures and result in a robust hyaline cartilage fill that ultimately improves the quality of life of patients with articular cartilage injuries.

\section{References}

1. Messner K, Maletius W (1996) The long-term prognosis for severe damage to weight-bearing cartilage in the knee: A 14-year clinical and radiographic follow-up in 28 young athletes. Acta Orthop Scand 67: 165-168.

2. Hunt HE, Sadr K, Deyoung AJ, et al. (2014) The role of immunologic response in fresh osteochondral allografting of the knee. Am J Sports Med 42: 886-891.
3. Shelbourne KD, Jari S, Gray T (2003) Outcome of untreated traumatic articular cartilage defects of the knee: A natural history study. J Bone Joint Surg Am 85: 8-16.

4. Abrams GD, Frank RM, Fortier LA, et al. (2013) Platelet-rich plasma for articular cartilage repair. Sports Med Arthrosc Rev 21: 213-219.

5. Abrams GD, Mall NA, Fortier LA, et al. (2013) BioCartilage: Background and operative technique. Operative Techniques in Sports Medicine 21: 116-124.

6. Frenkel SR, Di Cesare PE (1999) Degradation and repair of articular cartilage. Front Biosci 4: D671-D685.

7. Newman AP (1998) Articular cartilage repair. Am J Sports Med 26: 309-324.

8. Kleeblad L, van der List JP, Zuiderbaan HA, et al. (2017) Larger range of motion and increased return to activity, but higher revision rates following unicompartmental versus total knee arthroplasty in patients under 65: A systematic review. Knee Surg Sports Traumatol Arthrosc 26: 1811-1822.

9. Diduch DR, Insall JN, Scott WN, et al. (1997) Total knee replacement in young, active patients. Long-term follow-up and functional outcome. J Bone Joint Surg Am 79: 575-582.

10. Gioe TJ, Novak C, Sinner P, et al. (2007) Knee arthroplasty in the young patient: Survival in a community registry. Clin Orthop Relat Res 464: 83-87.

11. Goh GS, Liow MHL, Bin Abd Razak HR, et al. (2017) Patient-reported outcomes, quality of life, and satisfaction rates in young patients aged 50 years or younger after total knee arthroplasty. J Arthroplasty 32: 419-425.

12. Chen FS, Frenkel SR, Di Cesare PE (1999) Repair of articular cartilage defects: Part II. Treatment options. Am J Orthop (Belle Mead NJ) 28: 88-96.

13. Mithoefer K, McAdams T, Williams RJ, et al. (2009) Clinical efficacy of the microfracture technique for articular cartilage repair in the knee: An evidence-based systematic analysis. Am J Sports Med 37: 2053-2063.

14. Steadman JR, Rodkey WG, Briggs KK, et al. (1999) The microfracture technic in the management of complete cartilage defects in the knee joint. Orthopade 28: 26-32.

15. Mithoefer K, Williams RJ, Warren RF, et al. (2005) The microfracture technique for the treatment of articular cartilage lesions in the knee. A prospective cohort study. J Bone Joint Surg Am 87: 1911-1920.

16. Xing L, Jiang Y, Gui J, et al. (2013) Microfracture combined with osteochondral paste implantation was more effective than microfracture alone for full-thickness cartilage repair. Knee Surg Sports Traumatol Arthrosc 21: 1770-1776.

17. Knutsen G, Engebretsen L, Ludvigsen TC, et al. (2004) Autologous chondrocyte implantation compared with microfracture in the knee. A randomized trial. J Bone Joint Surg Am 86: 455-464.

18. Saris DB, Vanlauwe J, Victor J, et al. (2008) Characterized chondrocyte implantation results in better structural repair when treating symptomatic cartilage defects of the knee in a randomized controlled trial versus microfracture. Am J Sports Med 36: 235-246.

19. Nehrer S, Spector M, Minas T (1999) Histologic analysis of tissue after failed cartilage repair procedures. Clin Orthop Relat Res 1999: 149-162. 
20. Mithoefer K, Williams RJ, Warren RF, et al. (2006) High-impact athletics after knee articular cartilage repair: a prospective evaluation of the microfracture technique. Am J Sports Med 34: 1413-1418.

21. Hirahara AM, Mueller KW Jr (2015) BioCartilage: A new biomaterial to treat chondral lesions. Sports Med Arthrosc Rev 23: 143148.

22. Fortier LA, Chapman HS, Pownder SL, et al. (2016) BioCartilage improves cartilage repair compared with microfracture alone in an equine model of full-thickness cartilage loss. Am J Sports Med 44: $2366-2374$.

23. Chadha N, Sampson ESJ, Chen T, et al. (2012) Porous cartilage-derived matrix scaffolds for repair of articular cartilage defects. Orthopedic Research Society 2012 Annual Meeting.

24. Malinin T, Temple H, Carpenter E, et al. (2009) Induction of regeneration of articular cartilage defects by freeze-dried particulate cartilage allografts. Cartilage 1: 93-164.

25. Ashraf S, Han IB, Park H, et al. (2017) Role of RHEB in regulating differentiation fate of mesenchymal stem cells for cartilage and bone regeneration. Int J Mol Sci 18: 880 .

26. Pers YM, Rackwitz L, Ferreira R, et al. (2016) Adipose mesenchymal stromal cell-based therapy for severe osteoarthritis of the knee: A Phase I dose-escalation trial. Stem Cells Transl Med 5: 847-856.

27. Granéli C, Thorfve A, Ruetschi U, et al. (2014) Novel markers of osteogenic and adipogenic differentiation of human bone marrow stromal cells identified using a quantitative proteomics approach. Stem Cell Res 12: 153-165.

28. Basilio-de-Oliveira RP, Nunes Pannain VL (2015) Prognostic angiogenic markers (endoglin, VEGF, CD31) and tumor cell proliferation (Ki67) for gastrointestinal stromal tumors. World Journal of Gastroenterology 21: 6924-6930.
29. Hu K, Olsen BR (2016) The roles of vascular endothelial growth factor in bone repair and regeneration. Bone 91: 30-38.

30. Zelzer E, Mamluk R, Ferrara N, et al. (2004) VEGFA is necessary for chondrocyte survival during bone development. Development (Cambridge, England) 131: 2161-2171.

31. Lee CS, Burnsed OA, Raghuram V, et al. (2012) Adipose stem cells can secrete angiogenic factors that inhibit hyaline cartilage regeneration. Stem Cell Res Ther 3: 35.

32. (2019) A study to evaluate the efficacy of BioCartilage ${ }^{\circledR}$ micronized cartilage matrix in microfracture treatment of osteochondral defects. ClinicalTrials.gov.

33. (2019) Comparison of BioCartilage versus marrow stimulating procedure for cartilage defects of the knee. ClinicalTrials.gov.

34. Schallmo MS, Marquez-Lara A, Luo TD, et al. (2018) Arthroscopic treatment of hip chondral defect with microfracture and platelet-rich plasma-infused micronized cartilage allograft augmentation. Arthrosc Tech 7: e361-e365.

35. Carter AH, Guttierez N, Subhawong TK, et al. (2018) MR imaging of BioCartilage augmented microfracture surgery utilizing 2D MOCART and KOOS scores. J Clin Orthop Trauma 9: 146-152.

36. Clanton TO, Johnson NS, Matheny LM (2014) Use of cartilage extracellular matrix and bone marrow aspirate concentrate in treatment of osteochondral lesions of the talus. Techniques in Foot \& Ankle Surgery 13: 212-220.

37. Desai S (2014) Treatment of osteochondral lesions of the talus with marrow stimulation and micronized allograft cartilage matrix: An all-arthroscopic technique. Techniques in Foot \& Ankle Surgery 13: 167-173. 